\title{
Guest editorial: special issue on control of locomotion-from animals to robots
}

\author{
Auke Jan Ijspeert • Paolo Dario · Sten Grillner
}

Received: 25 February 2010 / Accepted: 25 February 2010 / Published online: 6 March 2010

(C) Springer Science+Business Media, LLC 2010

The locomotion skills of current robots are still far behind those of animals in terms of energy efficiency, flexibility, and agility in complex unstructured terrains. The goal of this special issue of Autonomous Robots is to capture aspects of the state of the art in locomotion control in biorobotics. These articles present interesting examples of interactions between biology and robotics. They demonstrate how inspiration from the animal kingdom can help to improve locomotion skills in robots, and/or illustrate how robots can be used as scientific tools in animal motor control, e.g. to test hypotheses about neural circuits and biomechanical principles in invertebrate and vertebrate animals.

The special issue covers different types of locomotion including swimming (Seo, Chung, and Slotine), snake-like crawling (Hatton and Choset; $\mathrm{Wu}$ and $\mathrm{Ma}$ ), jumping (Kovac, Schlegel, Zufferey, and Floreano; Hosoda, Sakaguchi, Takayama and Takuma), quadruped walking (Maufroy, Kimura, and Takase), and biped walking (Iida and Tedrake; Mombaur, Truong, and Laumond).

The first article by Seo and colleagues presents a swimming turtle robot that is controlled by a central pattern generator (CPG) model composed of a set of coupled oscillators. An interesting contribution of this article is that the oscillators are tightly linked to the mechanics and interact directly through the actual states of the actuators (as opposed to the

\footnotetext{
A.J. Ijspeert ( $\square)$

Ecole Polytechnique Fédérale de Lausanne, EPFL, Lausanne, Switzerland

e-mail: auke.ijspeert@epfl.ch

P. Dario

Scuola Superiore Sant'Anna, SSSA, Pisa, Italy

S. Grillner

Karolinska Institute, Stockholm, Sweden
}

desired states). This ensures well synchronized swimming gaits, and robustness against perturbations.

Hatton and Choset address the problem of gait generation for snake robots with many degrees of freedom. They propose a new approach for generating rolling and sidewinding gaits that combine ideas from two different approaches traditionally used in snake robotics, namely geometrical approaches and joint angle control approaches. An alternative approach based on CPGs is proposed by Wu and Ma. A new network made of Matsuoka oscillators with a feedback connection is proposed, which can generate uniform outputs without any additional adjustment.

A novel jumping robot is presented by Kovac and colleagues. The robot can make impressively high jumps. It has the interesting feature that it is capable of recovering passively after landing, orienting itself, and then to jump again. Jumping is also addressed by Hosoda and colleagues using muscle-like pneumatic actuators. Using inspiration from the vertebrate musculo-skeletal system, they designed a monopod robot that successfully uses bi-articular muscles for coordinating joints, and mono-articular ones for providing power for jumping. Pitti, Niiyama, and Kuniyoshi also use muscle-like actuators to explore the interplay between control, morphology and material. In particular, they present a control framework based on phase synchronization to implement neuro-modulators that can regulate the coordination between the body and the controllers' dynamics to different gait patterns, oscillatory but also discrete.

Maufroy and colleagues investigate the problem of posture control in quadruped locomotion. They consider locomotion at different speeds and present a control framework based on CPGs with the ability to integrate both posture and rhythmic motion controls and shift continuously from one control method to the other according to the walking speed. 
The special issue concludes with two articles on biped locomotion. Iida and Tedrake present a minimalistic control architecture for a simulated compass-like robot. They show that they can obtain basic walking stability without sensory feedback, not only on a flat terrain but also in various inclined slopes. Finally, Mombaur and colleagues apply inverse optimal control to establish a model of human path generation to given target positions and orientations. The approach is useful not only to identify the underlying optimality criteria of human motions but also to establish optimal control models that can be used to control the locomotion of the humanoid HRP2 robot. 